\title{
Magnetic Field Requirements for the CLAS12 Polarized Target
}

\section{Victoria Lagerquist ${ }^{* \dagger}$}

Old Dominion University

E-mail: vlage001@odu.edu

\begin{abstract}
Upcoming spin structure experiments in Hall B at Jefferson Lab will employ a new dynamically polarized target inside the CLAS12 detector system. Protons and deuterons in irradiated $\mathrm{NH}_{3}$ and $\mathrm{ND}_{3}$ will be polarized at $1 \mathrm{~K}$ using the $5 \mathrm{~T}$ field of the CLAS12 solenoidal magnet. For optimum polarization, the field uniformity requirements are around $100 \mathrm{ppm}$ over the volume of the target sample. I will discuss methods to improve field uniformity utilizing thin superconducting shim coils integrated within the $1 \mathrm{~K}$ refrigerator. I will also demonstrate that this method to adjust the $5 \mathrm{~T}$ field also enables the simultaneous opposite polarization of two adjacent target cells.
\end{abstract}

The 18th International Workshop on Polarized Sources, Targets, and Polarimetry, PSTP2019

23-27 September, 2019

Knoxville, Tennessee

\footnotetext{
* Speaker.

${ }^{\dagger}$ On behalf of the CLAS12 Polarized Target Collaboration.
} 


\section{Introduction}

The future physics program utilizing the upgraded CLAS12 detector [1] in Hall B of Jefferson Lab includes a series of experiments with a polarized target currently under construction by a collaboration between the Jefferson Lab Target Group, Old Dominion University, Christopher Newport University, and the University of Virginia. The target will utilize Dynamic Nuclear Polarization (DNP) to induce a net polarization in samples of protons $\left(\mathrm{NH}_{3}\right)$ and deuterons $\left(\mathrm{ND}_{3}\right)$ cooled to $1 \mathrm{~K}$ and in a $5 \mathrm{~T}$ field. The $1 \mathrm{~K}$ refrigerator is described elsewhere in these proceedings [2], while the field is generated by a $5 \mathrm{~T}$ superconducting solenoid that is part of the base CLAS12 detector package. [3].

In these proceedings we describe the design and modelling of superconducting correction, or shim, coils for the polarized target. These shims will be utilized to improve the field uniformity of the CLAS12 solenoid and optimize the target polarization. Alternatively, they can be used to modify the solenoid field such that two separate target samples can be simultaneously polarized in opposing directions with a single DNP microwave frequency.

\section{Field Requirements for Single and Double Targets}

The process of DNP requires a strong magnetic field with a relatively high degree of uniformity. As shown in the polarizing curves for $\mathrm{NH}_{3}$ and $\mathrm{ND}_{3}$ of Fig. 1, proton and deuteron polarizations of over $90 \%$ and approaching $50 \%$, respectively, can be achieved in frozen ammonia at a temperature of $1 \mathrm{~K}$ and a field of $5 \mathrm{~T}$. However, the curves also indicate that a variation of only $10^{-3}$ in $\Delta B / B$ will reduce the proton polarization by up to $20 \%$ and the deuteron polarization by as much as $50 \%$. For optimal polarization of these materials, a uniformity closer to $10^{-4}$ (100 ppm) over the volume of the target material is required. Preliminary results of the CLAS12 field map indicate a uniformity of about $300 \mathrm{ppm}$. Optimal polarization, especially in the case of the deuteron, will require additional magnetic coils to improve the field in the area of the target.

We are also investigating the simultaneous counter-polarization of adjacent target cells, allowing scattering measurements with both orientations simultaneously, and thus reducing certain systematic uncertainties. This technique has been previously utilized in polarized target experiments at CERN [5] [6] [7]. In those instances, the target samples were polarized using two microwave frequencies and a single value of the magnetic field. A sophisticated set of microwave absorbers and reflectors was utilized to contain each microwave frequency to its respective target cell. Alternatively, this operation can be performed with a single microwave frequency by adjusting the local fields around each target. As indicated by Fig. 1, the two fields need only differ by about $100 \mathrm{G}$. This eliminates the need for microwave containment, but requires careful modelling and construction of the shim coils used to manipulate the field for each cell.

\section{Shim Design and Simulation}

The design of shim coils for improving the field uniformity as well as enabling double-cell polarization was guided by an algorithm that minimizes the difference between the desired field for optimum single target DNP (or double polarization) and the field produced by the CLAS12 


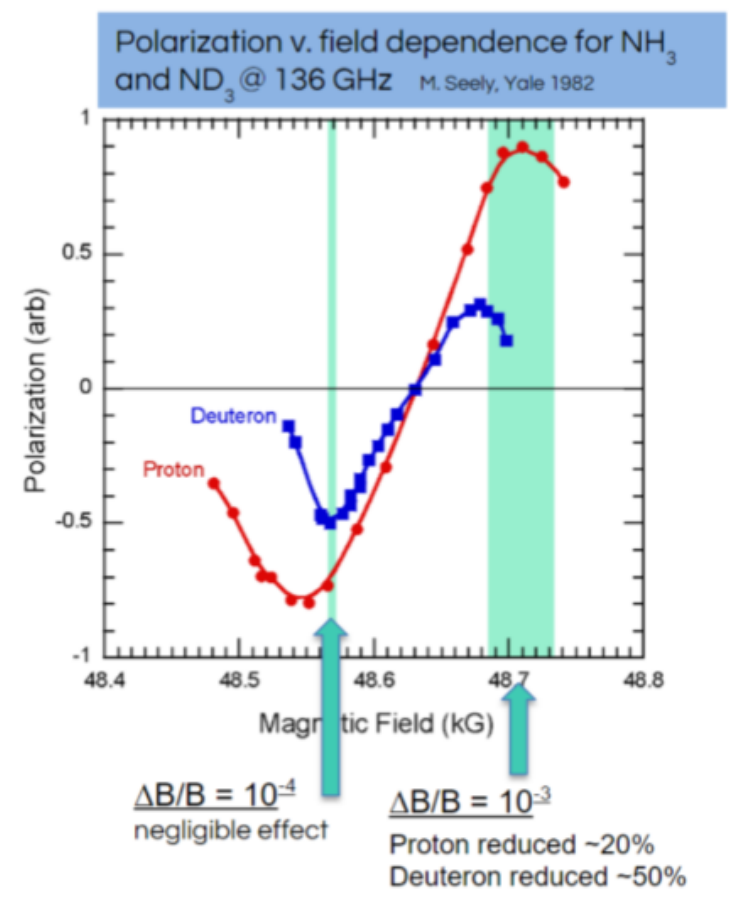

Figure 1: Polarization with respect to field value. [4]

solenoid. The biggest constraint on their design is the limited free volume available within the CLAS12 detector. In the target sample area, this is a $10 \mathrm{~cm}$ diameter cylindrical space. The majority of this space is taken up by the insulating vacuum chamber and thermal shielding for the target's $1 \mathrm{~K}$ refrigerator [2], leaving a working radius of approximately $2 \mathrm{~cm}$ for the coils.

Another constraint on the design of the coils is their ohmic heat load. Superconducting wire will be utilized for the coils themselves, but their normally conducting leads can represent a sizeable load on the warmer sections of the refrigerator. To minimize this, as well as to minimize the associated hardware, the design was confined to four total coils of minimal combined current, although variations with more and fewer coils were also simulated.

For the single target configuration, this constraint is straightforward. However, the double cell design requires a significant change in the field magnitude (up $160 \mathrm{G}$ ) over a relatively small axial distance $(2 \mathrm{~cm})$. To achieve these adjacent, independently uniform fields while minimizing the size, location, and current of the coils requires very careful coil design. Complicating the shim design, the optimal polarizing fields will vary with radiation dose from the beam, since the microwave frequency will remain constant. To that end, a program to optimize the necessary shim layout and currents is crucial.

Given the complexity of these requirements and the necessary degree of precision demanded of the fields, an algorithm was developed to optimize the shim coil arrangement. Written in MATLAB [8], the code takes as inputs the existing solenoid field profile and the final, desired field profile for a specified configuration of the target cell(s). It then varies the positions, widths, and currents of the shim coils (within specified restrictions), until a minimum difference between the calculated and desired fields is obtained. To address ohmic heating, the individual and total coil currents are 
included as weighting factors in determining the optimal design. Wire diameter, coil radius, and number of winding layers were specified for each simulation.

Simulations were made for optimizing the field uniformity to polarize single target cells or to generate the fields needed to polarize double cells in opposite directions. In the former case, cell lengths from 2 to $6 \mathrm{~cm}$ were examined. In the latter, studies were primarily confined to two, $2 \mathrm{~cm}$ long cells, separated by $2 \mathrm{~cm}$, although other configurations could be examined. Alternatively, the sizes and locations of the coils could be specified, and the optimum currents for achieving a different field profile could be determined.

\section{Test Results}

To test the success of the algorithm and to demonstrate the double-cell polarization technique, tests were conducted using prototype coils of normally-conducting copper and targets of two-part epoxy doped with the nitroxyl radical TEMPO [9]. For simplicity the tests were performed at $77 \mathrm{~K}$ using a warm-bore $5 \mathrm{~T}$ solenoid [10].

A coil design was chosen based on algorithm solutions emphasising low current due to the normal conducting wire. In other respects the prototype (wire gauge, coil diameter, etc) was constructed to satisfy the geometric requirements dictated by the $1 \mathrm{~K}$ refrigerator. Four coils of 32 AWG copper wire were wound onto an aluminum carrier (Fig. 2), with the location, widths, and number of layers determined by precision grooves machined in the carrier. The coils were fixed to the carrier using GE 7031 varnish. Parameters for the coils are given in Table 1 below.

\begin{tabular}{c|c|c|c} 
Coil & $\mathbf{z}(\mathbf{c m})$ & \# of Windings & Current (A) \\
\hline 1 & -3.5 & 132 & 0.940 \\
2 & -0.6 & 171 & 3.305 \\
3 & 0.6 & 172 & -3.284 \\
4 & 3.5 & 131 & -0.929
\end{tabular}

Table 1: Parameters of prototype coil set. For each coil, the inner radius is $2.7 \mathrm{~cm}$ with windings distributed between four layers.

The results, shown in Fig. 3, clearly demonstrate the efficacy of both the algorithm and the double-polarization technique. Although small, two NMR peaks of opposite polarization, obtained using a single NMR coil, are clearly visible. The microwave frequency was fixed approximately halfway between the normal frequencies for positive and negative polarizations. Additional details can be found in Ref [11].

\section{Conclusion}

In conclusion, the optimum magnetic field requirements for the CLAS12 Polarized Target appear to be easily achievable using small, superconducting coils integrated within the target cryostat. An algorithm for designing and operating these correction coils has been produced and successfully tested in a proof-of-principle demonstration at $77 \mathrm{~K}$. Superconducting NbTi coils will be wound and incorporated in the CLAS12 polarized target for testing at $1 \mathrm{~K}$ later this year. 


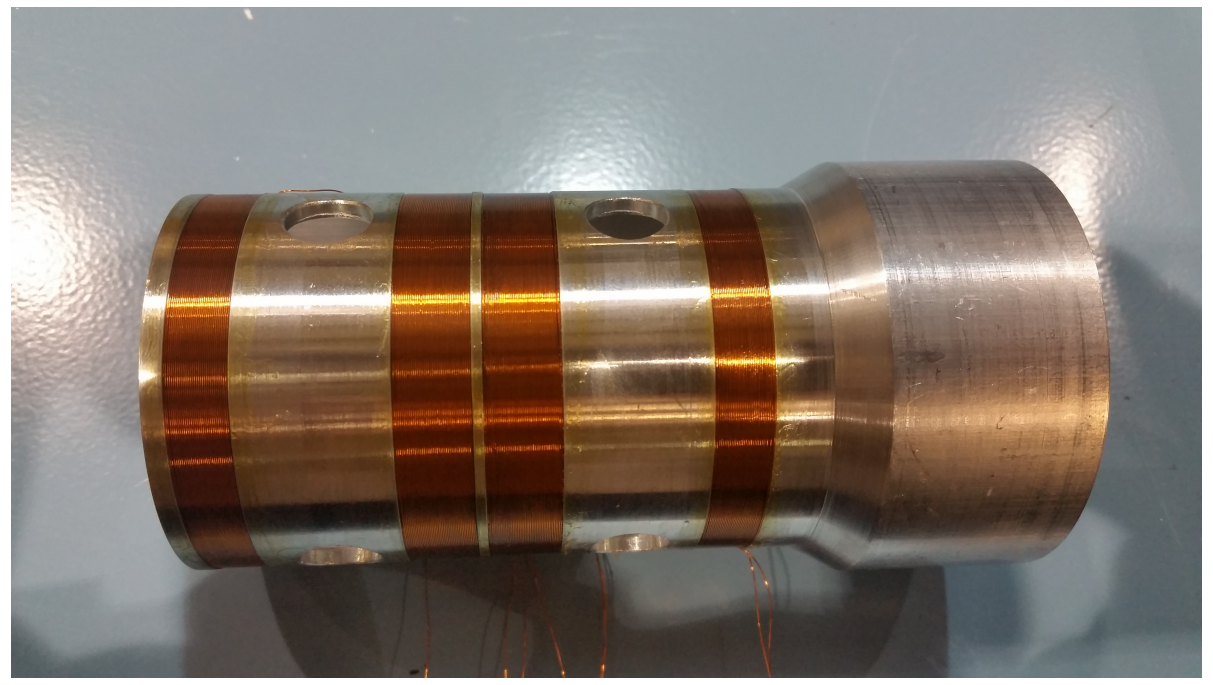

Figure 2: Shim coils on aluminum carrier.

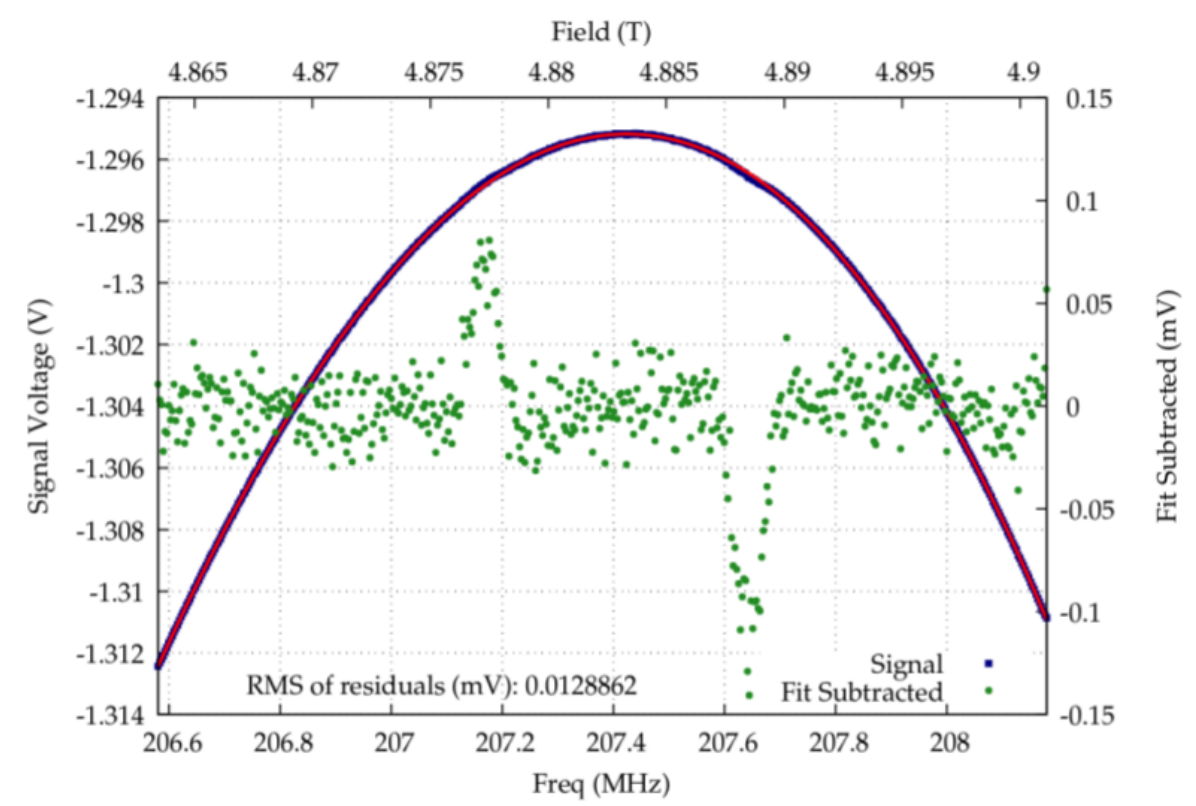

Figure 3: Polarization of two opposing cells.

\section{References}

[1] V. Burkert, et al. (2020) The CLAS12 spectrometer at Jefferson Laboratory. Nuclear Instruments and Methods in Physics Research. Section A: Accelerators, Spectrometers, Detectors, and Associated Equipment, 959, 163419. (doi: 10.1016/j.nima.2020.163419)

[2] J. Brock, these proceedings.

[3] R. Fair, et al. (2020) The CLAS12 superconducting magnets. Nuclear Instruments and Methods in Physics Research. Section A: Accelerators, Spectrometers, Detectors, and Associated Equipment, 952, 163578. (doi: 10.1016/j.nima.2020.163578) 
[4] M.L. Seely, PhD thesis, Yale University (1982).

[5] J. Ashman, et al. (1983) A measurement of the spin asymmetry and determination of the structure function g1 in deep inelastic muon-proton scattering. Physics Letters B, 206, 364 (doi: 10.1016/0370-2693(88)91523-7)

[6] D. Adams, et al. (1999) The polarized double cell target of the SMC. Nuclear Instruments and Methods in Physics Research. Section A: Accelerators, Spectrometers, Detectors, and Associated Equipment, 437, 23-67. (doi: 10.1016/S0168-9002(99)00582-3)

[7] J. Ball, et al. (2003) First results of the large COMPASS ${ }^{6} \mathrm{LiD}$ polarized target. Nuclear Instruments and Methods in Physics Research. Section A: Accelerators, Spectrometers, Detectors, and Associated Equipment, 498, 101-111. (doi: 10.1016/S0168-9002(02)02116-2)

[8] MATLAB. (2015). 8.5.0.197613 (R2015a). Natick, Massachusetts: The MathWorks Inc.

[9] Y. Noda, et al. (2015) Thermosetting polymer for dynamic nuclear polarization: Solidification of an epoxy resin mixture including TEMPO. Nuclear Instruments and Methods in Physics Research. Section A: Accelerators, Spectrometers, Detectors, and Associated Equipment, 776, 8-14. (doi: 10.1016/j.nima.2014.11.114)

[10] C.D. Keith, et al. (2012) The Jefferson Lab frozen spin target. Nuclear Instruments and Methods in Physics Research. Section A: Accelerators, Spectrometers, Detectors, and Associated Equipment, 674, 27. (doi: 10.1016/j.nima.2012.04.067)

[11] J. Maxwell, NMR Measurements for Solid Polarized Targets at Jefferson Lab, in Proceedings of the 23rd International Spin Physics Symposium. (SPIN2018)., 2018, https://pos.sissa.it/346/102/pdf. 